\title{
Distributed Symbolic Model Checking for $\mu$-Calculus
}

\author{
Orna Grumberg $^{1}$, Tamir Heyman ${ }^{1,2}$, and Assaf Schuster ${ }^{1}$ \\ ${ }^{1}$ Computer Science Department, Technion, Haifa, Israel \\ ${ }^{2}$ IBM Haifa Research Laboratories, Haifa, Israel
}

\begin{abstract}
In this paper we propose a distributed symbolic algorithm for model checking of propositional $\mu$-calculus formulas. $\mu$-calculus is a powerful formalism and many problems like (fair) CTL and LTL model checking can be solved using the $\mu$-calculus model checking. Previous works on distributed symbolic model checking were restricted to reachability analysis and safety properties. This work thus significantly extends the scope of properties that can be verified for very large designs.

The algorithm distributively evaluates subformulas. It results in sets of states which are evenly distributed among the processes. We show that this algorithm is scalable, and thus can be implemented on huge distributed clusters of computing nodes. In this way, the memory modules of the computing nodes collaborate to create a very large store, thus enables the checking of much larger designs. We formally prove the correctness of the parallel algorithm. We complement the distribution of the state sets by showing how to distribute the transition relation.
\end{abstract}

\section{Introduction}

In the early 1980's, model checking procedures have been suggested 51512 , which could handle systems with few thousands states. In the early 1990's, symbolic model checking methods have been introduced. These methods, based on Binary Decision Diagrams (BDDs) 2, could verify systems with $10^{20}$ states and more 4 . This progress has made model checking applicable to industrial designs of medium size. Significant efforts have been made since to fight the state explosion problem. But the need in verifying larger systems grows faster than the capacity of any newly developed method.

Recently, a new promising method for increasing the memory capacity was introduced. The method uses the collective pool of memory modules in a network of processes. In $\mid 10$, distributed symbolic reachability analysis has been performed, for finding the set of all states reachable from the initial states. In I , a distributed symbolic on-the-fly algorithm has been applied in order to model check properties written as regular expression. Experimental results show that distributed methods can achieve an average memory scale-up of 300 on 500 processes. Consequently, they find errors that were not found by sequential tools.

This paper extends the scope of properties that can be verified for large designs, by presenting a distributed symbolic model checking for the $\mu$-calculus. The $\mu$-calculus is a powerful formalism for expressing properties of transition systems using fixpoint operators. Many verification procedures can be solved by translating them into $\mu$-calculus model checking 4. Such problems include (fair) CTL model checking, LTL model checking, bisimulation equivalence and language containment of $\omega$-regular automata. 
Many algorithms for $\mu$-calculus model checking have been suggested $\mid 91618713$. In this work we parallelize a simple sequential algorithm, as presented in 6 . The algorithm works bottom-up through the formula, evaluating each subformula based on the evaluation of its own subformulas. A formula is interpreted as the set of states in which it is true. Thus, for each $\mu$-calculus operation, the algorithm receives a set (or sets) of states and returns a new set of states.

The distributed algorithm follows the same lines as the sequential one, except that each process runs its own copy of the algorithm and each set of states is stored distributively among the processes. Every process owns a slice of the set, so that the disjunction of all slices contains the whole set. An operation is now performed on a set (or sets) of slices and returns a set of slices. At no point in the distributed algorithm a whole set is stored by a single process.

Distributed computation might be subtle for some operations. For instance, in order to evaluate a formula of the form $\neg g$, the set of states satisfying $g$ should be complemented. It is impossible to carry this operation locally by each process. Rather, each process sends the other processes the states they own, which are not in $g$ to the best of its knowledge. If none of the processes "knows" that a state is in $g$, then it is (distributively) decided to be in $\neg g$.

While performing an operation, a process may obtain states that are not owned by it. For instance, when evaluating the formula $\mathbf{E X} f$, a process will find the set of all predecessor of states in its slice for $f$. However, some of these predecessors may belong to the slice of another process. Therefore, the procedure exch is executed (in parallel) by all processes, and each process sends its non-owned states to their respective owner.

Keeping the memory requirements low is done through frequent calls to a memory balancing procedure. It ensures that each set is partitioned evenly among the processes. This ensures that the memory requirements, commonly proportional to the size of the manipulated set, are evenly distributed among the processes. However, this also requires different slicing functions for different sets. As a result, we may need to apply an operation to two sets that are sliced according to different partitions. In the case of conjunction, for instance, first the two sets should be re-sliced according to the same partition. Only then the processes apply conjunction to their individual slices.

Distributing the sets of states is only one facet of the problem. The transition relation also strongly influences the memory peaks that appear during the computation of pre-image (EX) operations. The pre-image operation has one of the highest memory requirements in model checking. Even when its final result is of tractable size, its intermediate results might explode the memory. We propose a scalable distributed method for the pre-image computation, including partitioning of the transition relation.

\section{Preliminaries}

\subsection{The Propositional $\mu$-Calculus}

Below we define the propositional $\mu$-calculus II]. We will not distinguish between a set of states and the boolean function that characterizes this set. By abuse of notation we will apply both set operations and boolean operations on sets and boolean functions. Let $A P$ be a set of atomic propositions and let $V A R=\left\{Q, Q_{1}, Q_{2}, \ldots\right\}$ be a set of relational variables. The $\mu$-calculus formulas are defined as follows: if $p \in A P$, then $p$ 
is a formula; a relational variable $Q \in V A R$ is a formula; if $f$ and $g$ are formulas, then $\neg f, f \wedge g, f \vee g, \mathbf{E X} f$ are formulas; if $Q \in V A R$ and $f$ is a formula, then $\mu Q . f$ and $\nu Q . f$ are formulas. $\mu$-calculus consists of the set of closed formulas, in which every relational variable $Q$ is within the scope of $\mu Q$ or $\nu Q$.

Formulas of the $\mu$-calculus are interpreted with respect to a transition system $M=$ ( $S t, R, L)$ where $S t$ is a nonempty and finite set of states; $R \subseteq S t \times S t$ is the transition relation, and $L: S t \rightarrow 2^{A P}$ is the labelling function that maps each state to the set of atomic propositions true in that state.

In order to define the semantics of $\mu$-calculus formulas, we use an environment $e: V A R \rightarrow 2^{S t}$, which associates with each relational variable a set of states from $M$.

Given a transition system $M$ and an environment $e$, the semantics of a formula $f$, denoted $[[f]]_{M} e$, is the set of states in which $f$ is true. We denote by $e[Q \leftarrow W]$ a new environment that is the same as $e$ except that $e[Q \leftarrow W](Q)=W$. The set $[[f]]_{M} e$ is defined recursively as follows (where $M$ is omitted when clear from the context).

- $[[p]] e=\{s \mid p \in L(s)\}$

- $[[Q]] e=e(Q)$

- $[[\neg g]] e=S t \backslash[[g]] e$

- $\left[\left[g_{1} \wedge g_{2}\right]\right] e=\left[\left[g_{1}\right]\right] e \cap\left[\left[g_{2}\right]\right] e$

- $\left[\left[g_{1} \vee g_{2}\right]\right] e=\left[\left[g_{1}\right]\right] e \cup\left[\left[g_{2}\right]\right] e$

- $[[\mathbf{E X} g]] e=\{s \mid \exists t[(s, t) \in R$ and $t \in[[g]] e]\}$

- $[[\mu Q . g]] e$ and $[[\nu Q . g]] e$ are the least and greatest fixpoints, respectively, of the predicate transformer $\tau: 2^{S t} \rightarrow 2^{S t}$ defined by: $\tau(W)=[[g]] e[Q \leftarrow W]$

Tarski [7] showed that least and greatest fixpoints always exist if $\tau$ is monotone. If $\tau$ is also continuous, then the least and greatest fixpoints of $\tau$ can be computed by $\cup_{i} \tau^{i}($ False $)$ and $\cap_{i} \tau^{i}($ True $)$, respectively. In 6 it is shown that if $M$ is finite then any monotone $\tau$ is also continuous.

In this paper we consider only monotone formulas. Since we consider only finite transition systems, they are also continuous. The function fixpt on the right-handside of Figure Il describes an algorithm for computing the least or greatest fixpoint, depending on the initialization of $Q_{v a l}$. If the parameter $I$ is False then the least fixpoint is computed. Otherwise, if $I=$ True, then the greatest fixpoint is computed.

Given a transition system $M$, an environment $e$, and a formula $f$ of the $\mu$-calculus, the model checking algorithm for $\mu$-calculus finds the set of states in $M$ that satisfy $f$. Figure II presents a sequential recursive algorithm for evaluating $\mu$-calculus formulas. For closed $\mu$-calculus formulas, the initial environment is irrelevant. The necessary environments are constructed during recursive applications of the ev function.

\subsection{Elements of Distributed Symbolic Model Checking}

Our distributed algorithm involves several basic elements that were developed in 10 . For completeness, we briefly mention these elements in this subsection.

intermediate results, are represented by BDDs. the algorithm execution, the sets of states obtained are partitioned among the processes. A set of window functions is used to define the partitioning, determining the slice that is stored (we say: owned) by each process.

Definition 1. [Complete Set of Window Functions] A window function is a boolean function that characterizes a subset of the state space. A set of window functions 


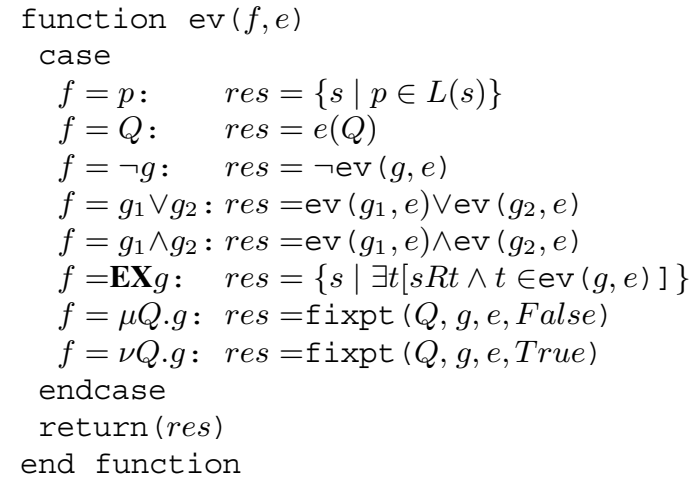

1 function fixpt $(Q, g, e, I)$

$Q_{v a l}=I$

repeat

$Q_{\text {old }}=Q_{\text {val }}$

$Q_{v a l}=\operatorname{ev}\left(g, e\left[Q \leftarrow Q_{o l d}\right]\right)$

until $\left(Q_{\text {val }}=Q_{\text {old }}\right)$

return $Q_{v a l}$

end function

Fig. 1. Pseudo-code for Sequential $\mu$-Calculus Model Checking.

$W_{1}, \ldots, W_{k}$ is complete if and only if for every $1 \leq i, j \leq k, i \neq j, W_{i} \wedge W_{j}=0$ and $\bigvee_{i=1}^{k} W_{i}=1$.

Unless otherwise stated, we assume that all sets of window functions are complete.

We use the slicing algorithm, as described in $|\mathrm{I0}|$ to get a set of window functions. The objective of this algorithm is to distribute a given set evenly among the nodes. Its input is a set of states, and its output is a set of window functions. These functions slices the input set into subsets that are approximately of the same size.

Maintaining balanced memory requirement by the processes is done by means of a memory balance algorithm, as described in 10. When this algorithm is applied at an already sliced set of states, a new partitioning is computed, one that will balance the size of the subsets. The new partitioning is computed by pairing large slice of the set with small one and re-slicing their union. This algorithm defines a new set of window functions that will be used to produce further intermediate results.

During the memory balance algorithm, as well as during other parts of the distributed model checking algorithm, BDDs are shipped between the processes. The communication uses a compact and universal BDD representation, as described in 10 . Different variable order is allowed in the different processes.

\section{Distributed Model Checking for $\mu$-Calculus.}

The general idea of the distributed algorithm is as follows. The algorithm consists of two phases. The initial phase starts as the sequential algorithm, described in Section 2 l It terminates when the memory requirement reaches a given threshold. At this point, the distributed phase begins. In order to distribute the work among the processes, the state space is partitioned into several parts, using a slicing procedure. Throughout the distributed phase, each process owns one part of the state space for every set of states associated with a certain subformula. When computation of a subformula produces states owned by other processes, these states are sent out to the respective processes. A memory balancing mechanism is used to repartition imbalance sets of states which 
are produced during the computation. Distributed termination algorithm is used to announce global termination. In the rest of this section, we describe elements used by this algorithm.

\subsection{Switching from Initial to Distributed Computation}

When the initial phase terminates, several subformulas have already been evaluated and the sets of states associated with them are stored. In order to start the distributed phase, we slice the sets of states found so far and distribute the slices among the processes.

Each set of states is represented by a BDD and its size is measured by the number of BDD nodes. All sets are managed by the same BDD manager, where parts of the BDDs that are used by several sets are shared and stored only once. Thus, when partitioning the sets, there are two factors involved: the required storage space for the sets, and the space needed to manipulate them. In order to keep the first factor small, it is best to partition the sets so that the space used by the BDD manager for all sets in each process is small. To keep the second factor small, observe that the memory used in performing an operation is proportional to the size of the set it is applied to, thus the part of each set in each process should be small.

In model checking, the most acute peaks in memory requirement usually occur while operations are performed. Thus, it is more important to reduce the second factor. Indeed, rather than minimizing the total size of each process, our algorithm slices each set in a way that reduces the size of its parts. It is important to note that as a result the slicing criterion may differ for different sets.

We use a slicing algorithm 10 described generally in Section $\Sigma 2$ In order to slice all the sets that where already evaluated at the point of phase switching, slicing is applied to each one of them.

While the slicing algorithm works it updates two tables: InitEval and InitSet. InitEval keeps track of which sets have been evaluated by the initial phase of the algorithm. InitEval $(f)$ is True if and only if $f$ has been evaluated by the initial algorithm. Each process $i d$ has the table InitSet that for each formula $f$, holds the subset of the set of states satisfying $f$ and owned by this process. Formally, for each process $i d$, InitSet $(f)=f \wedge W_{i d}$. The distributed phase will start by sending the tables InitEval and InitSet and the list of slices $W_{i}$ to all the processes.

\subsection{The Distributed Phase}

The distributed version of the model checking algorithm for the $\mu$-calculus is given in Figure 2 While the sequential algorithm finds the set of states in a given model that satisfy a formula of the $\mu$-calculus logic, in the distributed algorithm each process finds the part of this set that the process owns. Intuitively, the distributed algorithm works as follows: given a set of slices $W_{i}$, a formula $f$, and an environment $e$, the process $i d$ finds the set of states $\operatorname{ev}(f, e) \wedge W_{i d}$.

In fact, a weaker property is required in order to guarantee the correctness of the algorithm. We only need to know that when evaluating a formula $f$, every state satisfying $f$ is collected by at least one of the processes. For efficiency, however, we require in addition that every state is collected by exactly one process. 
Given a formula $f$ the algorithm first checks if the initial phase has already evaluated it by checking if InitEval $(f)=$ True. If so, it uses the result stored in InitSet $(f)$. Otherwise, it evaluates the formula recursively. Each recursive application associates a set of states with some subformula.

Preserving the work load is an inherent problem in distributed computation. If the memory requirement in one of the processes is significantly larger than the others, then the effectiveness of the distributed system is destroyed. To avoid this situation, whenever a new set of states is created a memory balance procedure is invoked to keep a balanced memory requirement by the new set. The memory balance procedure changes the slices $W_{i}$ and updates the parts of the new set in each of the processes accordingly. Each process in the distributed algorithm evaluates each subformula $f$ as follow (see Figure 2:

A propositional formula $p \in A P$ : evaluated by collecting all the states $s$ that satisfy two conditions: $p$ is in the labelling $L(s)$ of $s$ and in addition $s$ is owned by this process.

A relational variable $Q$ : evaluated using the local environment of the process. Since only closed $\mu$-calculus formulas are evaluated, the environment must have a value for $Q$ (computed in a previous step).

A subformula of the form $\neg g$ : evaluated by first evaluating $g$, and then using the special function exchnot. Given a set of states $S$ and a partition $S_{1}, \ldots, S_{k}$ of $S$, each process $i$ runs the procedure exchnot on $S_{i}$. The process reports all other processes of the states that do not belong to $S$ "as far as it knows". Since each state in $S$ belongs to some process, if none of the processes knows that $s$ is in $S$, then $s$ is in $\neg S$.

Since each process holds only the states of $\neg S$ that it owns, the processes actually send each other only states that owned by the receiver. This reduces communication.

A subformula of the form $g_{1} \vee g_{2}$ : evaluated by first evaluating $g_{1}$ and $g_{2}$, possibly with different slicing functions. This means that a process can hold a part of $g_{1}$ with respect to one slicing and a part of $g_{2}$ with respect to another slicing. Nevertheless, since each state of $g_{1}$ and of $g_{2}$ belongs to one of the processes, each state of $g_{1} \vee g_{2}$ now belongs to one of the processes. Applying the function exch results in a correct distribution of the states among the processes, according to the current slicing.

A subformula of the form $g_{1} \wedge g_{2}$ can be translated using De Morgan's laws to $\neg\left(\neg g_{1} \vee \neg g_{2}\right)$. However, evaluating the translated formula requires four communication phases (via exch and exchnot). Instead, such a formula is evaluated by first evaluating $g_{1}$ and $g_{2}$. As in the previous case, they might be evaluated with respect to different window functions. Here, however, the slicing of the two formulas should agree before a conjunction can be applied. This is achieved by applying exch twice, thus the overall communication is reduced to only two rounds.

A subformula of the form $\mathbf{E X} g$ : evaluated by first evaluating $g$ and then computing the pre-image using the transition relation $R$. Since every state of $g$ belongs to one of the processes, every state of the pre-image also belongs to one. In fact, a state may be computed by more than one process if it is obtained as a pre-image of two parts. Applying exch completes the evaluation correctly.

Subformulas of the form $\mu Q . g$ and $\nu Q . g$ (the least fixpoint and greatest fixpoint, respectively): evaluated using a special function fixpt that iterates until a fixpoint is found. The computations for the formulas differ only in the initialization which is False for $\mu Q . g$ and the current window functions for $\nu Q . g$. 


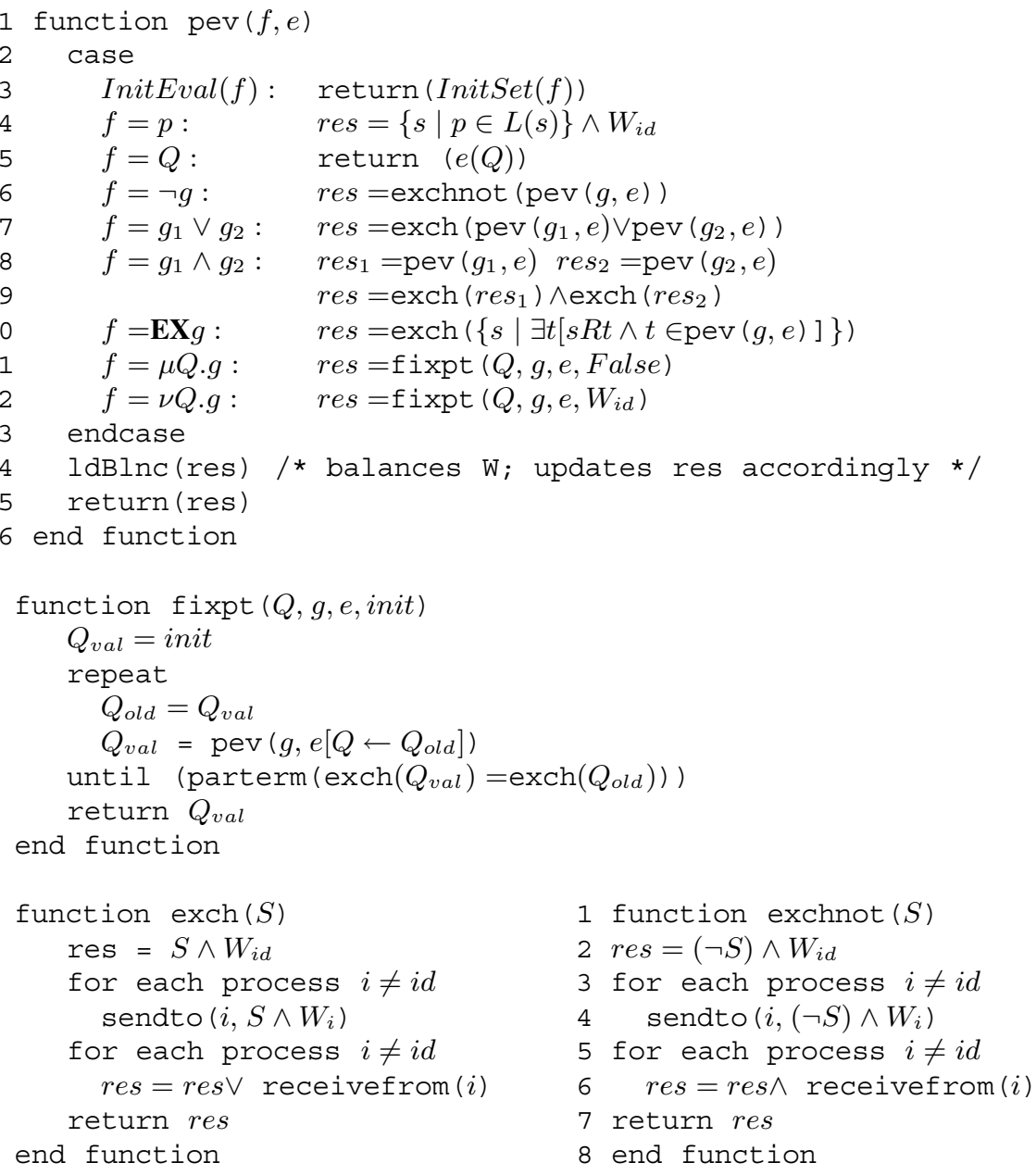

Fig. 2. Pseudo-code for a Process $i d$ in the Distributed Model Checking

\subsection{Sources of Scalability}

The efficiency of a parallelization approach is determined by the ratio between computation complexity, normalized by computation speed, and communication complexity, normalized by communication bandwidth. In our parallel model checking algorithm, this ratio (excluding normalization, which is dependent on the underlying platform) can be estimated by observing that peak memory requirement for a single $\mu$-calculus operation of a symbolic computation is a lower bound on the computation complexity of this operation. On average, in the distributed setup, the size of BDD structures that are sent (received) by a process is a fraction of its BDD manager size at the end of the operation (after memory balance). Thus, roughly speaking, for a single operation computation, peak memory utilization bounds from below the computation complexity, whereas the 
size of the BDD manager represents the communication complexity. General wisdom holds that the ratio between peak and manager sizes reaches 2 or 3 orders of magnitudes, which, for current computing platforms is sufficient to keep the processor and communication subsystems equally busy. Indeed, our experiments with previous parallel symbolic computations in a distributed setup consisting of a slow network confirmed the efficiency of this approach $10 \mathrm{II}$.

Scalability of a parallel system is the ability to include more processes in order to handle larger inputs of higher complexity. Linear scalability is used to describe a parallel system that does not loose performance while scaling up. Recall that the volume of communication performed by a single process in our algorithm during a single operation, may be represented on average by a fraction of its BDD manager size at the end of the operation. Also, the corresponding peak memory that is used by the process during that operation is bounded by the size of its memory module (otherwise the operation overflows). By the above mentioned ratio between the sizes of the peak and the BDD manager, the manager size (in between operations) is also bounded. Thus, using our effective slicing procedure, the local BDD manager size does not increase when the system is scaled up globally in order to check larger models using more processes. Thus, the ratio between computation and communication for each process does not vary substantially when the system scales up, implying almost linear scalability of our distributed model checking algorithm.

Finally, we note that a higher ratio of peak to BDD manager sizes, which may result from a larger transition system in larger models, will enhance the scalability of our parallel approach. Since the size of memory module limits the peak size, a higher ratio implies smaller BDD manager, which, in turn, implies lower communication volumes. Thus, when the checked models grow, the method may exhibit super-linear scalability.

\section{Correctness}

In this section we prove the correctness of the distributed algorithm, assuming the sequential algorithm is correct. The sequential algorithm evaluates a formula by computing the set of states satisfying this formula. In the distributed algorithm every such set is partitioned among the processes. The union over all the partitions for a given subformula is called the global set. In the proof we show that, for every $\mu$-calculus formula, the set of states computed by the sequential algorithm is identical to the global set computed by the distributed algorithm. Note that, the global set is never actually computed and is introduced only for the sake of the correctness proof. In the proof that follows we need the following definition.

Definition 2. [Well Partitioned Environment] An environment e is well partitioned by parts $e_{1}, \ldots, e_{k}$ if and only if, for every $Q \in V A R, e(Q)=\bigvee_{i=1}^{k} e_{i}(Q)$.

The procedures exch are applied by all processes with a set of non-disjoint subsets $S_{i}$ that cover a set res. Given a set of window functions, the procedures exchange nonowned parts so that at termination each process has all the states from res it owns. The set of window functions do not change.

Let $f$ be a $\mu$-calculus formula, $e_{i d}$ be the environment in process $i d \cdot \operatorname{pev}_{i d}\left(f, e_{i d}\right)$ denotes the set of states returned by procedure pev, when run by process $i d$ on $f$ and $e_{i d}$. 
Theorem II defines the relationship between the outputs of the sequential and the distributed algorithms.

Theorem 1 (Correctness). Let $f$ be a $\mu$-calculus formula, e be a well partitioned environment by $e_{1}, \ldots e_{k}, e^{\prime}$ be the environment when ev $(f, e)$ terminates and for all $i=1, \ldots, k, e_{i}^{\prime}$ be the environment when $\operatorname{pev}_{i}\left(f, e_{i}\right)$ terminates. Then, $e^{\prime}$ is well partitioned by $e_{1}^{\prime}, \ldots e_{k}^{\prime}$ and $\mathrm{ev}(f, e)=\bigvee_{i=1}^{k} \operatorname{pev}_{i}\left(f, e_{i}\right)$.

Proof: We prove the theorem by induction on the structure of $f$. In all but the last two cases of the induction step the environments are not changed and therefore $e^{\prime}$ is well partitioned by $e_{1}^{\prime}, \ldots e_{k}^{\prime}$. Due to lack of space we only consider several of the more interesting cases.

Base: $f=p$ for $p \in A P$ - Immediate.

\section{Induction:}

$\underline{f=Q}$, where $Q \in V A R$ is a relational variable: $\bigvee_{i=1}^{k} \operatorname{pev}_{i}\left(Q, e_{i}\right)=\bigvee_{i=1}^{k} e_{i}(Q)$. $\overline{\text { Since } e}$ is well partitioned, $e(Q)=\bigvee_{i=1}^{k} e_{i}(Q)$, which is equal to ev $(f, e)$.

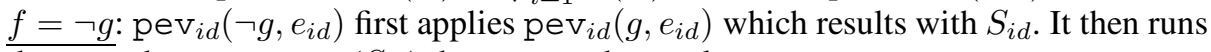
the procedure exchnot $\left(S_{i d}\right)$ that returns the result $r e s_{i d}$.

$$
r e s_{i d}=\left(\left(\neg S_{i d}\right) \wedge W_{i d}\right) \wedge \bigwedge_{j \neq i d}\left(\left(\neg S_{j}\right) \wedge W_{i d}\right)=\bigwedge_{j=1}^{k}\left(\left(\neg S_{j}\right) \wedge W_{i d}\right) .
$$

When exchnot terminates in all processes, the global set computed by all processes is (recall that $\bigvee_{i=1}^{k} W_{i}=1$ ):

$$
\bigvee_{i=1}^{k}\left(\bigwedge_{j=1}^{k}\left(\left(\neg S_{j}\right) \wedge W_{i}\right)\right)=\bigwedge_{j=1}^{k}\left(\neg S_{j}\right) \wedge \bigvee_{i=1}^{k} W_{i}=\bigwedge_{j=1}^{k}\left(\neg S_{j}\right)=\neg \bigvee_{j=1}^{k} S_{j}
$$

Since $S_{i}=\operatorname{pev}_{i}\left(g, e_{i}\right), \neg \bigvee_{j=1}^{k} S_{j}=\neg \bigvee_{j=1}^{k} \operatorname{pev}_{i}\left(g, e_{i}\right)$, which by the induction hypothesis is identical to $\neg \operatorname{ev}(g, e)$. This, in turn, is identical to $\operatorname{ev}(\neg g, e)$. Applying $\mathrm{IdBlnc}$ at the end of pev, repartitions the subsets between the processes, however, their disjunction remains the same. Thus, ev $(\neg g, e)=\bigvee_{i=1}^{k} \operatorname{pev}_{i}\left(\neg g, e_{i}\right)$.

$f=g_{1} \vee g_{2}: \operatorname{pev}_{i d}\left(f, e_{i d}\right)$ first computes pevid $\left(g_{1}, e_{i d}\right) \vee \operatorname{pev}_{i d}\left(g_{2}, e_{i d}\right)$. At the end of this computation, the global set is:

$$
\bigvee_{i=1}^{k}\left(\operatorname{pev}_{i}\left(g_{1}, e_{i}\right) \vee \operatorname{pev}_{i}\left(g_{2}, e_{i}\right)\right)=\bigvee_{i=1}^{k} \operatorname{pev}_{i}\left(g_{1}, e_{i}\right) \vee \bigvee_{i=1}^{k} \operatorname{pev}_{i}\left(g_{2}, e_{i}\right)
$$

By the induction hypothesis, this is identical to ev $\left(g_{1}, e\right) \vee$ ev $\left(g_{2}, e\right)$ which is identical to ev $\left(g_{1} \vee g_{2}, e\right)$. Applying the procedures exch and ldBlnc change the partition of the sets among the processes, but not the global set.

$f=\mu Q . g$, a least fixpoint formula: As in previous cases, we would like to prove that $\bar{\bigvee}_{i=1}^{k} \operatorname{pev}_{i}\left(\mu Q . g, e_{i}\right)=\operatorname{ev}(\mu Q . g, e)$. Since $1 \mathrm{dBlnc}$ does not change the correctness of this claim, we only need to prove that $\bigvee_{i=1}^{k} \mathrm{fixpt}_{i}\left(Q, g, e_{i}\right.$, False $\left.)\right)=$ fixpt $(Q, g, e, F$ alse $))$. In addition, we need to show that the environment remains well partitioned when the computation terminates. The following lemma proves stronger requirements. The lemma uses the following property of procedure parterm. 
Property 1. Procedure parterm is invoked by each of the processes with a boolean parameter. If all parameters are True, then parterm returns True to all processes. Otherwise, it returns False to all processes.

Lemma 1. Let $Q^{j}$, be the value of $Q_{\text {val }}$ in iteration $j$ of the sequential fixpoint algorithm. Similarly, let $Q_{i d}^{j}$ be the value of $Q_{v a l}$ in iteration $j$ of the distributed fixpoint algorithm in process $i d . Q^{0}$ is the initialization of the sequential algorithm; $Q_{i d}^{0}$ is the initialization of the distributed algorithm. Then, - In every iteration, $e$ is well partitioned by $e_{1}, \ldots, e_{k}$. For every $j: Q^{j}=\bigvee_{i=1}^{k} Q_{i}^{j}$. If the sequential fixpt algorithm terminates after $i_{0}$ iterations then so does the distributed fixpoint algorithm.

Proof: We prove the lemma by induction on the number $j$ of iterations in the loop of the sequential function $\mathrm{f}$ ixpt.

Base: $j=0$ :

- At iteration $0, e$ is well partitioned based on the induction hypothesis of Theorem II

- In case $f=\mu Q . g$, the initialization of the sequential algorithm, as well as the distributed algorithm is False. Hence, $Q^{0}=Q_{i d}^{0}=$ False which implies $Q^{0}=\bigvee_{i=1}^{k} Q_{i}^{0}$. - Both algorithms perform at least one iteration, so they do not terminate at iteration 0 . Induction: Assume Lemma II holds for iteration $j$. We prove it for iteration $j+1$.

- Let $e^{\prime}, e_{1}^{\prime}, \ldots, e_{k}^{\prime}$ be the environments at the end of iteration $j+1$, and assume that $e$ is well partitioned by $e_{1}, \ldots, e_{k}$ at the end of iteration $j$. The only changes to the environments in iteration $j+1$ may occur in line 5 of the distributed and sequential algorithms. In the sequential algorithm $e$ may be changed in two ways: $e(Q)$ is assigned a new value $Q^{j}$, or a recursive call to ev may change $e$. Similarly, in the distributed algorithm two changes may occur: $e_{i d}(Q)$ is assigned a new value $Q_{i d}^{j}$, or a recursive call to pevid may change $e_{i d}$.

By the induction hypothesis of Lemma II we know that $Q^{j}=\bigvee_{i=1}^{k} Q_{i}^{j}$, hence $e\left[Q \leftarrow Q^{j}\right](Q)=\bigvee_{i=1}^{k} e_{i}\left[Q \leftarrow Q_{i}^{j}\right](Q)$. Since no other change has been made to the environments, and since $e$ is well partitioned, we conclude that $e\left[Q \leftarrow Q^{j}\right]$ is well partitioned by $e_{1}\left[Q \leftarrow Q_{1}^{j}\right], \ldots, e_{k}\left[Q \leftarrow Q_{k}^{j}\right]$.

In iteration $j+1$, ev in now invoked with an environment that is well partitioned by the environments pev $_{i d}$ is invoked with. The induction hypothesis of Theorem 1 therefore guarantees that $e^{\prime}$ is well partitioned by $e_{1}^{\prime}, \ldots, e_{k}^{\prime}$.

- $Q^{j+1}=\operatorname{ev}\left(g, e\left[Q \leftarrow Q^{j}\right]\right)$ (line 5 of the sequential algorithm) and $Q_{i d}^{j+1}=$ $\operatorname{pev}_{i d}\left(g, e\left[Q \leftarrow Q_{i d}^{j}\right]\right)$ (line 5 of the distributed algorithm).

By the first bullet above, $e\left[Q \leftarrow Q^{j}\right]$ is well partitioned. Thus, the induction hypothesis of Theorem $\mathbb{I}$ is applicable and implies that ev $\left(g, e\left[Q \leftarrow Q^{j}\right]\right)=$ $\bigvee_{i=1}^{k} \operatorname{pev}_{i}\left(g, e\left[Q \leftarrow Q_{i}^{j}\right]\right)$. Hence, $Q^{j+1}=\bigvee_{i=1}^{k} Q_{i}^{j+1}$.

- The sequential fixpt procedure terminates at iteration $j+1$ if $Q^{j}=Q^{j+1}$. We prove that this holds if and only if for every process $i d, \operatorname{exch}\left(Q_{i d}^{j}\right)=\operatorname{exch}\left(Q_{i d}^{j+1}\right)$ and therefore parterm returns True to all processes.

Let $W_{1}, \ldots, W_{k}$ be the current window functions. By the second bullet above, $Q^{j}=$ $\bigvee_{i=1}^{k} Q_{i}^{j}$ and $Q^{j+1}=\bigvee_{i=1}^{k} Q_{i}^{j+1}$.

$$
\forall i d\left[\operatorname{exch}\left(Q_{i d}^{j}\right)=\operatorname{exch}\left(Q_{i d}^{j+1}\right)\right] \Leftrightarrow \forall i d\left[\bigvee_{i=1}^{k} Q_{i}^{j} \wedge W_{i d}=\bigvee_{i=1}^{k} Q_{i}^{j+1} \wedge W_{i d}\right] \Leftrightarrow
$$




$$
\forall i d\left[Q^{j} \wedge W_{i d}=Q^{j+1} \wedge W_{i d}\right] \Leftrightarrow Q^{j}=Q^{j+1} .
$$

The last equality is implied by the previous one since the window functions are complete. This complete the proof of the lemma and also the proof of the theorem. Q.E.D.

The above theorem can be extended to state that when all procedures pevid $\left(f, e_{i d}\right)$ terminate, the subsets owned by each of the processes are disjoint. This is important in order to avoid duplication of work. However, it is not necessary for the correctness of the model checking algorithm.

\section{Scalable Distributed Pre-image Computation}

The main goal of our distributed algorithm is to reduce the memory requirement. In symbolic model checking, pre-image is one of the operations with the highest memory requirement. Given a set of states $S$, pre-image computes $\operatorname{pred}(S)$ (also denoted by EX $S$ in $\mu$-calculus), which is the set of all predecessors of states in $S$. The pre-image operation can be described by the formula $\operatorname{pred}(S)=\exists s^{\prime}\left[R\left(s, s^{\prime}\right) \wedge S\left(s^{\prime}\right)\right]$. It is easy to see that the memory requirement of this operation grows with the sizes of the transition relation $R$ and the set $S$. Furthermore, intermediate results sometimes exceed the memory capacity even when $\operatorname{pred}(S)$ can be held in memory.

Our distributed algorithm reduces memory requirements by slicing each of the computed sets of states. This takes care of the $S$ parameter of pre-image, but not of $R$. In order to make our method scalable for very large models, we need to reduce the size of the transition relation as well.

The transition relation consists of pairs of states. We distinguish between the source states and the target states by refer to the latter as $S t^{\prime}$. Thus, $R \subseteq S t \times S t^{\prime}$.

A reduction of the second parameter of $R, S t^{\prime}$, can be achieved by applying the well-known restriction operator 8 : Prior to any application of pre-image, a process that owns a slice $S_{i}$ of $S$ reduces its copy of $R$ by restricting $S t^{\prime}$ to $S_{i}$. This reduction is dynamic since pre-image operations are applied to different sets during model checking.

We further reduce $R$ by adding a static slicing of $S t$ according to (possibly different) window functions $U_{1}, \ldots, U_{m}$. The slicing algorithm of Section 2.2 can be used to produce $U_{1}, \ldots, U_{m}$, so that $R$ is partitioned to $m$ slices of similar size. Each slice $R_{j}$ is a subset of $\left(S t \cap U_{j}\right) \times S t^{\prime}$. Since $R$ does not change during the computation, $U_{1}, \ldots, U_{m}$ do not change as well.

Having $k$ window functions $W_{1}, \ldots, W_{k}$ for $S$ and $m$ window functions $U_{1}, \ldots, U_{m}$ for $R$, we use $k$ groups of $m$ processes each. All processes in the same group have the same $W_{i}$, and hence own the same $S_{i}=S \cap W_{i}$. However, each process in the group has a different $U_{j}$. Process $(i, j)$ with $W_{i}$ and $U_{j}$ computes pre-image of $S_{i}$ by $\operatorname{pred}_{j}\left(S_{i}\right)=\exists s^{\prime}\left[R_{j}\left(s, s^{\prime}\right) \wedge S_{i}\left(s^{\prime}\right)\right]$. Since $U_{1}, \ldots, U_{m}$ is a complete set of window functions, $\bigvee_{j=1}^{m} \operatorname{pred}_{j}\left(S_{i}\right)=\operatorname{pred}\left(S_{i}\right)$. Thus, the group with window function $W_{i}$ computes the same set as process $i$ in the algorithm of Section $\mathbf{B}$

Once the computation is completed, procedure exch is applied to exchange nonowned states (according to $W_{i}$ ). Procedure $1 \mathrm{dBlnc}$ is used to update the $W_{i}$ window functions in order to balance the memory load. Both procedures are defined as before. However, when $l \mathrm{dBlnc}$ changes the window functions, all members in each of the groups should agree on the new window function. 


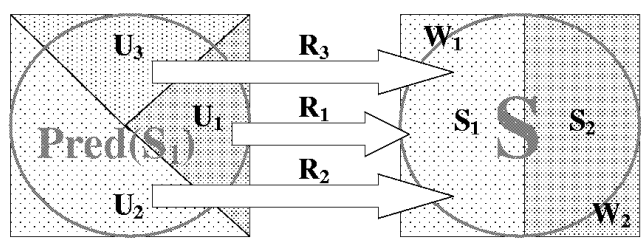

The Figure above demonstrates a pre-image computation using sliced transition relation with $k=2$ and $m=3$. Given a set $S$ sliced into $S_{1}, S_{2}$ according to $W_{1}, W_{2}$ respectively, the pre-image of $S_{1}$ is computed by three processes. Each process uses a different slice of the transition relation, $R_{1}, R_{2}$ and $R_{3}$, according to $U_{1}, U_{2}$ and $U_{3}$.

The method suggested in this section applies slicing to the full transition relation in case it can be held in memory, but is too large to enable a successful completion of the pre-image operation. However, often the transition relation is given partitioned, i.e., given as a set of small relations $N_{l}$, each defining the value of variable $v_{l}$ in the next states. The size of the partitioned transition relation is usually small, therefore can be constructed by one process and then be sliced using the algorithm suggested in 14 . In this case the model checking is done directly with the partitioned transition relation 3 .

\subsection{Distributed Construction of the Sliced Full Transition Relation}

The full transition relation $R$ is a conjunction of all $N_{l}$. Here we consider cases where either $R$ or its construction cannot fit into the memory of a single process.

Our goal is to construct slices $R_{j}$ of $R$, with none of the processes ever holding $R$. Each process starts constructing by gradually conjuncting partitions $N_{l}$, until a threshold is reached. The current (partial) transition relation is then partitioned among the processes, using the slicing algorithm. Each process continues to conjunct the partitions that have not been handled yet, until all partitions are conjuncted. During conjunction, further slicing or balancing are applied so that the final slices will be balanced.

\section{Acknowledgement}

We would like to thank Ken McMillan for his time and patient and for his help in choosing a notation for the $\mu$-calculus model checking algorithm.

\section{References}

1. S. Ben-David, T. Heyman, O. Grumberg, and A. Schuster. Scalable Distributed On-theFly Symbolic Model Checking. In Third International Conference on Formal methods in Computer-Aided Design (FMCAD'O0), Austin, Texas, November 2000.

2. R. E. Bryant. Graph-based Algorithms for Boolean Function Manipulation. IEEE Transactions on Computers, C-35(8):677-691, 1986.

3. J. R. Burch, E. M. Clarke, and D. E. Long. Symbolic Model Checking with Partitioned Transition Relations. In A. Halaas and P. B. Denyer, editors, Proceedings of the 1991 International Conference on Very Large Scale Integration, August 1991.

4. J. R. Burch, E. M. Clarke, K. L. McMillan, D. L. Dill, and L. J. Hwang. Symbolic Model Checking: $10^{20}$ States and Beyond. Information and Computation, 98(2):142-170, 1992. 
5. E. M. Clarke, E. A. Emerson, and A. P. Sistla. Automatic verification of finite-state concurrent systems using temporal logic specifications. In Proceedings of the Tenth Annual ACM Symposium on Principles of Programming Languages, January 1983.

6. E.M. Clarke, O. Grumberg, and D.A. Peled. Model Checking. MIT press, December 1999.

7. R. Cleaveland. Tableau-based model checking in the propositional mu-calculus. Acta Informatica, 27:725-747, 1990.

8. O. Coudert, J. C. Madre, and C. Berthet. Verifying of Synchronous Sequential Machines Based on Symbolic Execution. In J. Sifakis, editor, Workshop on Automatic Verification Methods for Finite State Systems, pages 365-373. Springer-Verlag, Grenoble, France, 1989.

9. E. A. Emerson and C.-L. Lei. Efficient Model Checking in Fragments of the Propositional Mu-calculus. In Proceedings of the First Annual Symposium on Logic in Computer Science. IEEE Computer Society Press, June 1986.

10. T. Heyman, D. Geist, O. Grumberg, and A. Schuster. Achieving Scalability in Parallel Reachability Analysis of Very Large Circuits. In Proc. of the 12th International Conference on Computer Aided Verification. Springer-Verlag, June 2000.

11. D. Kozen. Results on the propositional $\mu$-calculus. TCS, 27, 1983.

12. O. Lichtenstein and A. Pnueli. Checking that finite state concurrent programs satisfy their linear specification. In Proceedings of the Twelfth Annual ACM Symposium on Principles of Programming Languages, pages 97-107, January 1985.

13. D. Long, A. Browne, E. Clark, S. jha, and W. Marrero. An Improved Algorithm for the Evaluation of Fixpoint Expressions. In Proc. of the Sixth International Conference on Computer Aided Verification, LNCS 818, pages 338-350. Springer-Verlag, 1994.

14. A. Narayan, A. Isles, J. Jain, R. Brayton, and A. L. Sangiovanni-Vincentelli. Reachability Analysis Using Partitioned-ROBDDs. In Proceedings of the IEEE International Conference on Computer Aided Design, pages 388-393. IEEE Computer Society Press, June 1997.

15. J.P. Quielle and J. Sifakis. Specification and verification of concurrent systems in CESAR. In Proceedings of the Fifth International Symposium in Programming, 1981.

16. C. Stirling and D. J. Walker. Local Model Checking in the Model Mu-Calculus. In Proc. of the 1989 Int. Joint Conf. on Theory and Practice of Software Development, 1989.

17. A. Tarski. A lattice-theoretical fixpoint theorem and its applications. Pacific J. Math, 1955.

18. G. Winskel. Model checking in the modal $\nu$-calculus. In Proceedings of the Sixteenth International Colloquium on Automata, Languages, and Programming, 1989. 